\title{
MODIfICACIÓN DEL ABORDAJE DE CALDWELL-LUC PARA EL APRENDIZAJE DE LA ANATOMÍA DE LA ÓRBITA
}

\author{
Rodríguez Vargas, Gabriel y Guevara Arroyo, Victoria
}

Departamento de Anatomía de la Escuela de Medicina de la Universidad de Costa Rica, San Pedro, San José, Costa Rica

Resumen: El abordaje anatómico clásico para la disección de la órbita se realiza de manera transcranial, dificultando la movilización y visualización completa del contenido total de la órbita. Mediante un abordaje transmaxilar con remoción del piso de la órbita y una cantolísis bilateral; con la ayuda de un equipo de disección básico, una herramienta rotatoria de alta velocidad y lentes de magnificación, el Departamento de Anatomía de la Universidad de Costa Rica presenta en este estudio una nueva propuesta para la visualización detallada de todas las estructuras anatómicas contenidas en la órbita, mediante una modificación de la técnica de Caldwell-Luc. Este abordaje, debe únicamente utilizarse para el estudio académico de la órbita y no como técnica quirúrgica debido a las complicaciones ya conocidas que proveen los abordajes transmaxilares abiertos como el de Caldwell-Luc a los pacientes.

Palabras clave: órbita, disección transmaxilar, anatomía 


\title{
MODIFICATION OF CALDWELL-LUC'S APPROACH FOR THE ANATOMIC LEARNING OF THE ORBIT
}

\begin{abstract}
Classically, orbit dissection approach is done trans cranially, hindering mobilization and complete visualization of the total content of the orbit. By a transmaxillary approach with orbital floor removal and bilateral cantholysis, performed with just a basic dissection kit, a high speed rotation tool and magnifying lenses, the Anatomy Department of the University of Costa Rica presents in this study a new proposal for detailed visualization of all the anatomical structures contained within the orbit, based on a modification of the Caldwell-Luc surgical technique. This approach must be reserved only for the academic study of the orbit and not as a surgical technique due to the complications already known that opened transmaxilary approaches, such as Caldwell-Luc's provide to patients.
\end{abstract}

Key words: orbit, transmaxilar dissection, anatomy

\section{INTRODUCCIÓN}

La anatomía del contenido de la órbita es bastante compleja ya que describe numerosas estructuras musculares, nerviosas y vasculares dentro de un volumen muy pequeño [1]. El abordaje académico para la disección de la órbita se realiza de manera tradicional por un abordaje transcranial, a través de la fosa craneal anterior [2]. Esto presenta el inconveniente de que requiere realizar craneotomías para poder observar las estructuras y limita la observación de estructuras de la pared medial, lateral y del piso de la órbita [3].

Por otro lado, los abordajes quirúrgicos actuales de la órbita varían dependiendo de la región de la misma a la cual se intente acceder [3]. En el caso especifico de las fracturas del piso de la órbita, inicialmente se realizaba el abordaje de antrostomía Caldwell-Luc, descrita en 1972 por Walter [4]. Hoy en día, los abordajes transconjuntival y subciliar, presentan menos complicaciones que la antrostomía (entropión, ectropión en $<5 \%$ de los pacientes) permitiendo una adecuada visualización del piso de la órbita; y junto con la endoscopia maxilar, han dejado la antrostomía casi en el olvido en nuestros días como procedimiento quirúrgico, debido a sus múltiples y no tan infrecuentes complicaciones (anestesia o parestesias infraorbitarios, sinusitis a repetición, sinequias nasales y complicaciones dentales) $[5,15]$.

En el 2003, Gonül y colaboradores describieron un estudio anatómico para la visualización a través del seno maxilar, de las estructuras cercanas al piso de la órbita y las estructuras intraconales utilizando el abordaje de Caldwell-Luc, retomando la técnica pero enfocado hacia el aprendizaje anatómico [6].

En este articulo, el Departamento de Anatomía de la Facultad de Medicina de la Universidad de Costa Rica, posterior a una revisión topográfica de la anatomía orbitario, proponemos un abordaje que permita visualizar la totalidad de las estructuras intraorbitarias mediante una modificación a la técnica de Caldwell-Luc, realizando una disección transmaxilar de la órbita asociado a cantolísis bilateral. Este abordaje puede facilitar el estudio de la anatomía de la órbita enormemente y simplificar la disección de la zona, permitiendo observar una mayor gama de estructuras con respecto al abordaje transcranial clásico y al propuesto por Gonül. 


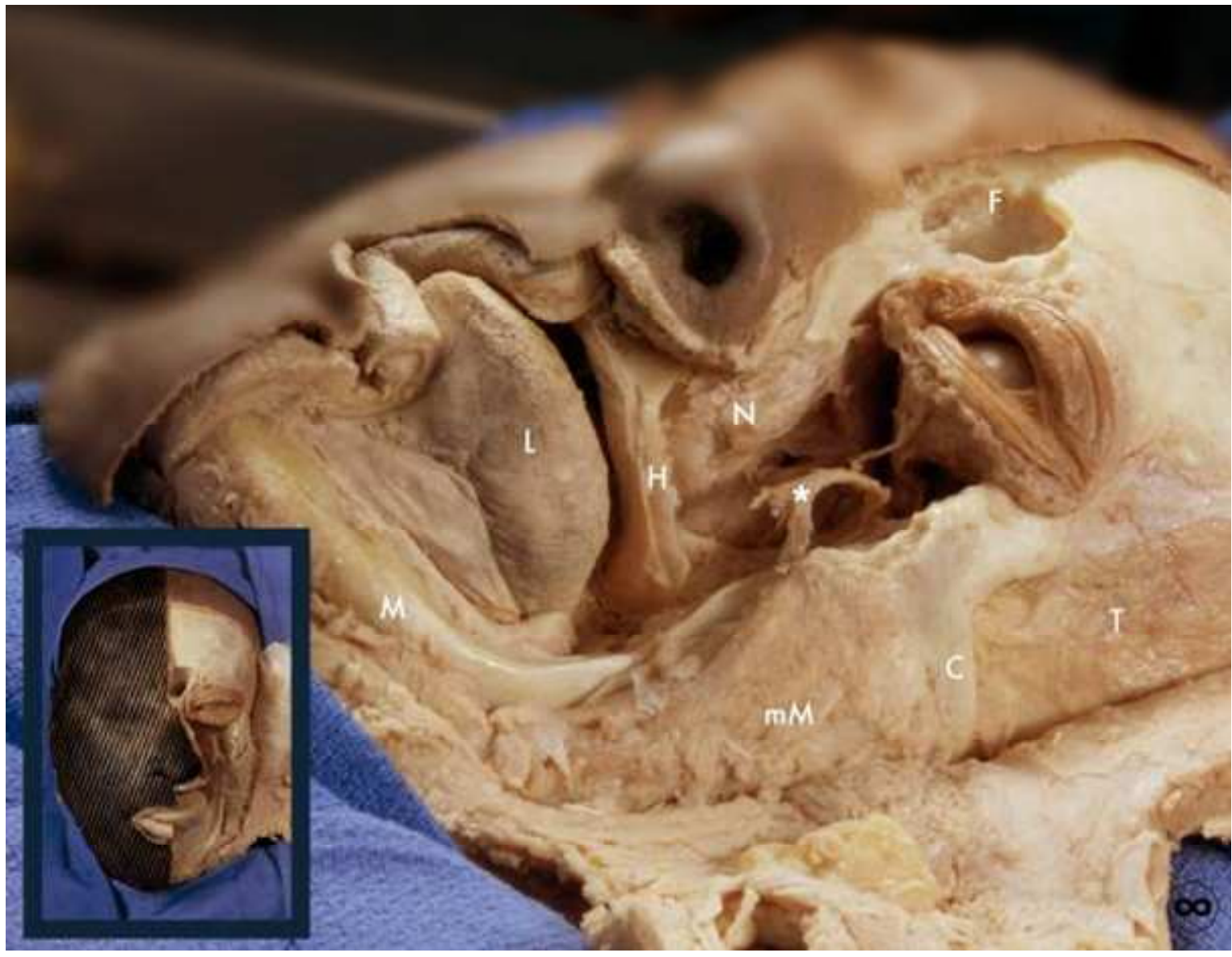

Figura 1. Vista Panorámica de la Disección Transmaxilar de la Órbita. La piel y el tejido celular subcutáneo fueron disecados hacia lateral dejando expuestos: La Lengua (L), el Cuerpo de la Mandíbula(M), el músculo Masetero (mM), el Arco Cigomático (C), y el músculo Temporal (T).Además, el antro del hueso maxilar ha sido removido manteniendo únicamente la Base del Hueso Maxilar (H), lo que permite visualizar la Pared Nasal Lateral $(N)$ y el Nervio Infraorbitario (*); este es el espacio al cual se desplazará posteriormente el contenido orbitario. Obsérvese en el recuadro inferior izquierdo la incisión realizada sobre la piel desde una vista anterior. Seno Frontal (F)

\section{MATERIALES Y MÉTODOS}

Para este estudio se utilizó un cadáver humano previamente fijado, un equipo de disección básico, lentes de magnificación y una herramienta rotatoria de alta velocidad (Dremel ${ }^{\circledR}$ ) para el corte de los huesos. Todas las fotografías fueron tomadas con una cámara Canon $^{\circledR}$ EOS 7D con un lente Canon ${ }^{\circledR}$ EF100mm f/2.8 Macro y otro Canon 28-135mm f/3.5-5.6 IS.

\section{TÉCNICA DE DISECCIÓN}

Se realizó una incisión en la piel a nivel de la línea media anterior de la cara que se continuó sobre la base de la pirámide nasal (Figura 1). La misma se continuó por el borde de la mandíbula y por la inserción del cuero cabelludo. Además se realizó una incisión sobre el borde orbitario. Se reflejaron la piel, el tejido celular subcutáneo junto a las estructuras superficiales y la capa muscular hacia lateral en un sólo bloque continuando las mismas incisiones previamente realizadas. Se ejecutó una antrostomía estándar de Caldwell-Luc, sin incidir más allá de la apófisis cigomática del hueso maxilar. El piso de la órbita fue removido desde el borde orbitario hasta el ápex del

Revista electrónica publicada por el Departamento de Farmacología de la Escuela de Medicina de la Universidad de Costa Rica, 2060 San José, Costa Rica. ${ }^{\circledR}$ All rights reserved. Licensed under a Creative Commons Unported License. 
seno maxilar, manteniendo especial cuidado en esta etapa para no lesionar el nervio infraorbitario. Ambos cortes en hueso se realizan con la herramienta rotativa de alta velocidad. Se procedió a continuación a disecar el piso de la órbita removiendo el periostio orbitario y la grasa periorbitaria visible a este nivel, posteriormente se realizó la cantolísis bilateral para poder desplazar el contenido orbitario en un solo bloque hacia el seno maxilar para completar la disección de las estructuras craneales al meridiano orbitario removiendo la grasa orbitaria residual. Esta última etapa requiere la utilización de lentes de magnificación de ser posible debido a que las estructuras intraorbitarios son pequeñas y frágiles.

\section{RESULTADOS}

Al ingresar al seno maxilar luego de realizar la antrostomía, es posible identificar de manera apropiada el nervio infraorbitario discurriendo por debajo del piso de la órbita, este debe de ser separado del agujero infraorbitario, junto con su arteria, para poder separar la pared anterior del hueso maxilar y obtener acceso libre al seno maxilar.

Posteriormente, al eliminar el piso de la órbita, las primeras dos estructuras en visualizarse fueron los músculos recto inferior y recto medial, así como el oblicuo inferior. Además si se realiza un sondaje del conducto nasolagrimal a partir del punto lagrimal inferior, es posible observar el trayecto del conducto nasolagrimal hasta su entrada en la nariz a través del meato inferior. En este punto es posible realizar dos abordajes para ingresar al cono muscular de la órbita; el medial, desplazando el recto inferior hacia lateral y el lateral, al desplazar el recto inferior hacia medial. Por el abordaje medial se puede observar la división inferior del nervio oculomotor, mientras que por el abordaje lateral se puede observar el ganglio ciliar adherido al nervio óptico, así como se puede observar parte del drenaje venoso de la órbita por el plexo pterigoideo (Figura 2).

Una vez realizada la cantolísis de manera bilateral, se liberaron los puntos de soporte para el globo ocular en la órbita, esto permite fácilmente desplazar el contenido orbitario hacia el espacio ocupado por el seno maxilar, dando la vista superior de la órbita, que permitió la visualización de los músculos

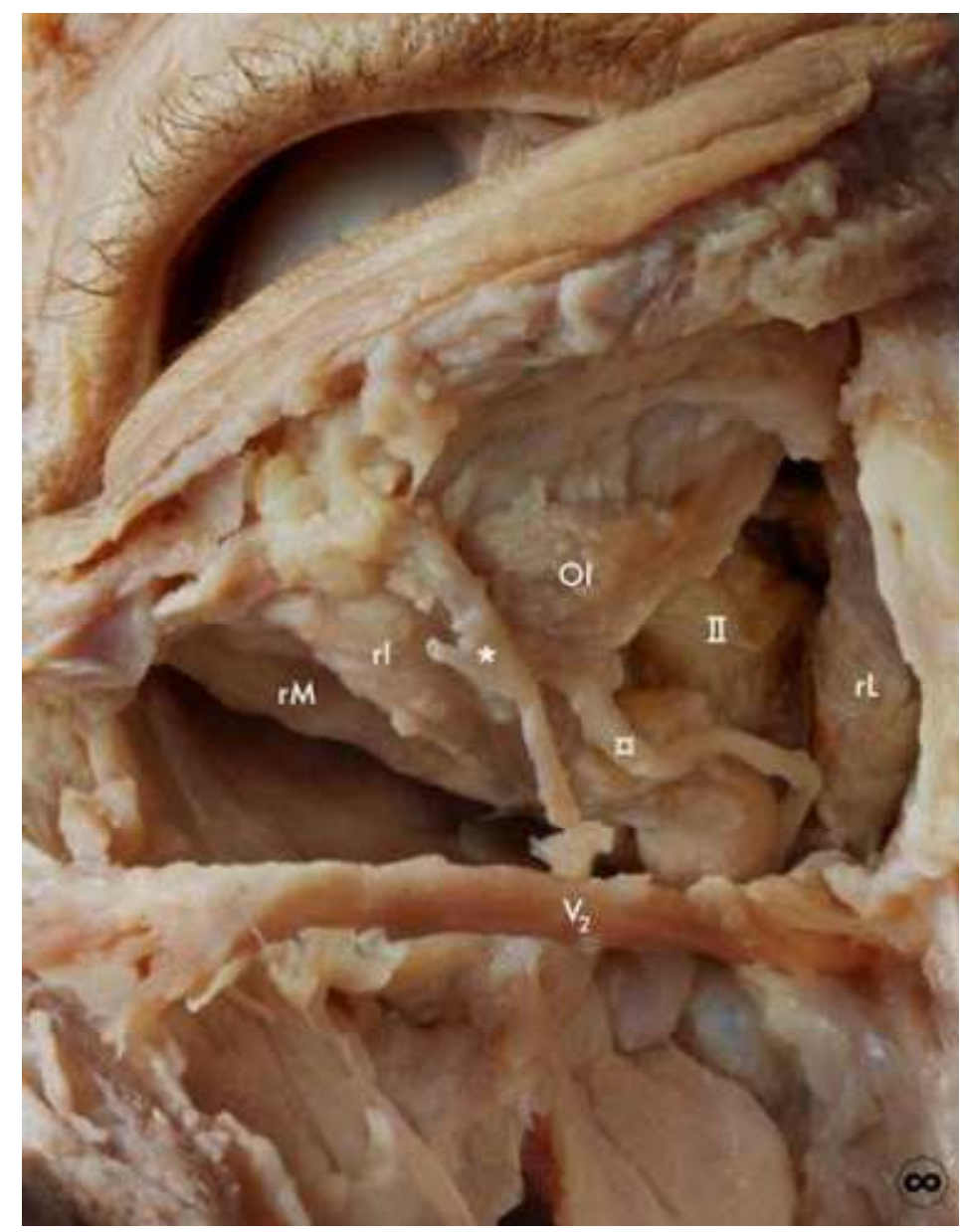

Figura 2. Vista Inferior de la Órbita. Mediante este abordaje se pueden visualizar los músculos: Recto Medial (rM), Recto Inferior ( $\mathrm{rl}$ ), Oblicuo Inferior (OI) y Recto Lateral ( $\mathrm{rL}$ ). También se observan la división inferior de la arteria oftálmica (x), el Nervio Óptico (II) y el Nervio Infraorbitario $\left(\mathrm{V}_{2}\right)$ con la rama palpebral que envía este mismo $\left(^{*}\right)$

recto superior, oblicuo superior y elevador del párpado. Además de la visualización de las arterias supraorbitarios y supraciliares que acompañan a los nervios de mismo nombre, los cuales provienen del nervio frontal, rama de la primera porción del trigémino. Todo este grupo vasculo nervioso se encuentra adherido al periostio del techo orbitario. De la misma manera se puede observar justo por encima y hacia lateral de la aponeurosis del elevador, la glándula lagrimal con la arteria lagrimal a su lado, mientras a nivel medial se puede observar como las fibras del músculo oblicuo superior modifican su orientación al llegar a la tróclea para orientarse hacia su sitio de inserción postecuatorial en el globo ocular (Figura 3). 


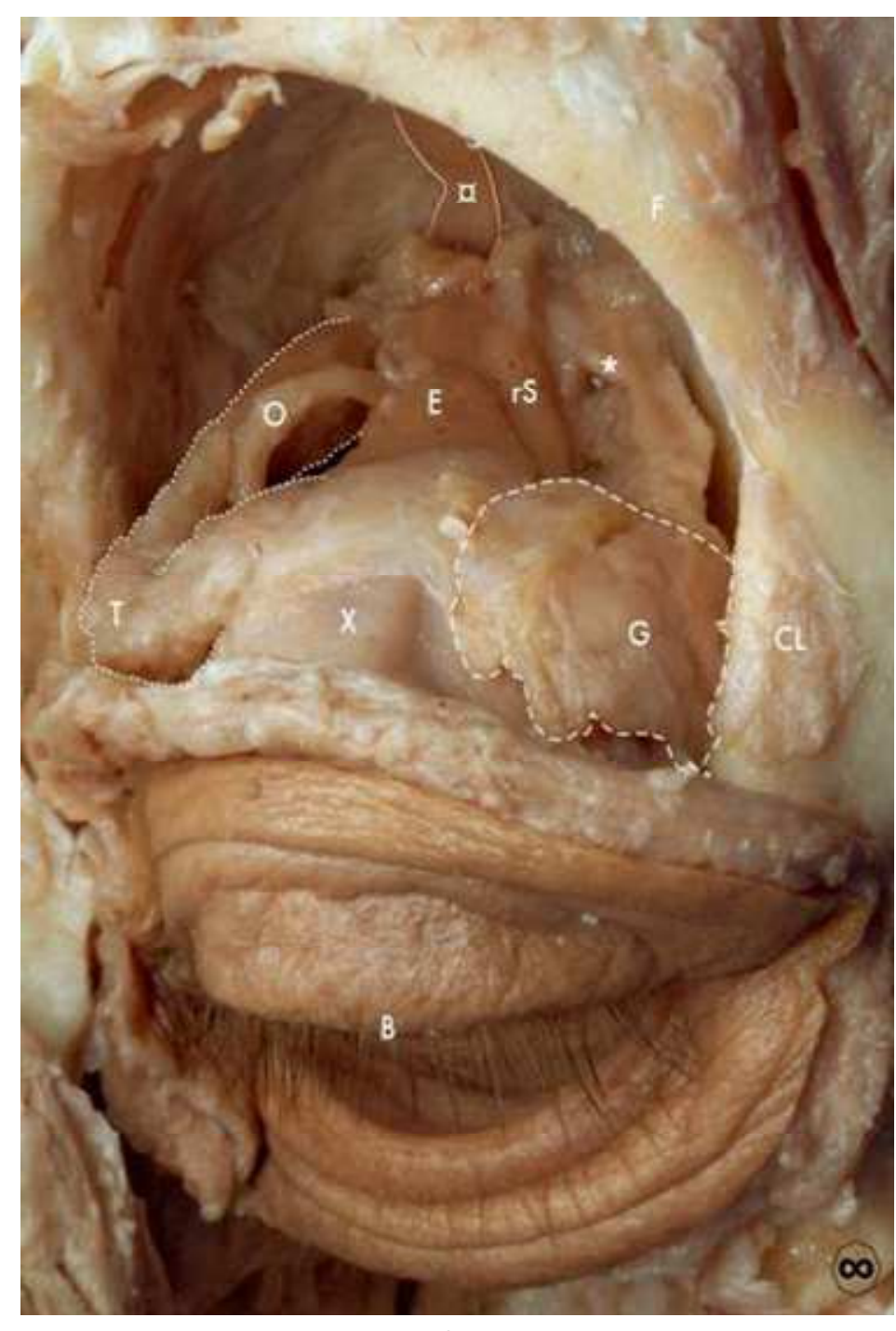

Figura 3. Vista Superior de la Órbita.

Se observan los músculos Oblicuo Superior (………) con la Tróclea del mismo (T), Elevador del Párpado Superior (E) cubriendo parcialmente en la imagen almúsculo Recto superior (rS). Además, La Glándula Lagrimal (G, ------), la Capsula de Tenon (X), la Arteria Oftálmica (O), la Arteria Lagrimal $\left({ }^{*}\right)$ y el paquete vasculo-nervioso que contiene al Nervio Frontal y las arterias supratroclear y supraorbitaria ( $x$, $\longrightarrow$ ) se observan dentro de la órbita. Hueso Frontal (F), Borde palpebral superior (B) Sitio de inserción del Canto Lateral (CL).

Si se realiza una ligera rotación interna del globo ocular, se puede observar el músculo recto lateral, y si se realiza rotación externa, se puede observar el músculo recto medial. Las fibras del músculo orbicular del ojo son visibles alrededor de la incisión periorbitaria.

\section{DISCUSIÓN}

El abordaje transcraneal a través del techo de la órbita, no permite una adecuada visualización de todas las estructuras que se encuentran dentro de la órbita, ya que imposibilita la visualización del músculo recto inferior o el músculo oblicuo inferior, por ejemplo; inclusive este abordaje sólo permite una visualización parcial de la glándula lagrimal y de los músculos recto medial y recto lateral [1].

Además, los abordajes quirúrgicos de la órbita hoy en día intentan ser lo menos invasivos posibles, como lo son el abordaje subciliar y transconjuntival, debido a los beneficios que esto representa para el paciente (menor tiempo de recuperación, menor índice de complicaciones, mejores resultados estéticos] [3, 6],y junto a los abordajes endoscópicos que representan una estrategia aún menos invasiva, en manos experimentadas dan excelentes resultados para el manejo de las patologías de la órbita [7].

Estos abordajes, sin embargo, no permiten la valoración completa de la anatomía orbitario, ya que son enfocados al área donde se encuentra la patología específica y dificultan claramente el aprendizaje de la anatomía orbitario para los estudiantes, médicos y especialistas en formación [8].

El abordaje propuesto, que realiza una antrostomía de Caldwell-Luc[9] en la cual se modifica la incisión del surco bucogingival a una incisión en piel y añade una cantolísis bilateral ofrece múltiples ventajas académicas, para quien requiera estudiar de manera más profunda la anatomía orbitario, ya que permite visualizar más estructuras y de manera más completa.

Se puede mediante este abordaje visualizar: todos los músculos extraoculares con su origen e inserción; todas las estructuras que se encuentran dentro del cono muscular, que incluye la inervación, irrigación y drenaje de la órbita y el ojo. La vista inferior permite observar el trayecto del conducto nasolagrimal, el seno maxilar completo y el nervio infraorbitario; mientras que la vista superior visualiza la glándula lagrimal completa y la tróclea del músculo oblicuo superior.

Revista electrónica publicada por el Departamento de Farmacología de la Escuela de Medicina de la Universidad de Costa Rica, 2060 San José, Costa Rica. ${ }^{\circledR}$ All rights reserved. Licensed under a Creative Commons Unported License. 
Todo esto se da gracias a que al eliminar el piso de la órbita y realizar la cantolísis, se permite una mayor movilización del contenido orbitario, al poderlo desplazar parcialmente hacia el seno maxilar. Es posible inclusive realizar cierto grado de rotación interna y externa de las estructuras, para maniobrar entre las mismas, lo cual permite realizar una mejor comprensión de la anatomía de este espacio y de las relaciones que guardan sus estructuras dentro del mismo, favoreciendo que los estudiantes comprendan de mejor manera su funcionamiento. Este abordaje presenta además la ventaja de que se realiza sin la necesidad de realizar una craneotomía para poder acceder a la órbita, disminuyendo el tiempo de disección y aumentando el área de trabajo.

Sin embargo, este abordaje propuesto, también presenta una serie de inconvenientes. Dentro de los más notables es que evidentemente este abordaje es solamente un abordaje anatómico, mas no quirúrgico, ya que el compromiso sobre las estructuras óseas maxilares, la cantolísis y la manipulación excesiva del globo ocular y tejido intraorbitario comprometerían gravemente la cara y probablemente el ojo del paciente, por lo que debe reservarse este abordaje únicamente para el proceso académico. Por otro lado, para realizar la disección, se recomienda el uso de lentes de aumento para lograr preservar las estructuras durante el proceso de disección sin lesionarlas. Es necesario además realizar una disección a nivel de la superficie de la cara que podría comprometer ciertas estructuras, principalmente de algunos músculos de la expresión facial, como el músculo elevador del labio superior, el músculo del ala de la nariz y el músculo cigomático menor, ya que es necesario desperiostizar al menos parcialmente la zona en la cual éstos se originan a nivel del antro maxilar. Y por último, a pesar de que se permite una gran maniobrabilidad de las estructuras, es importante manipular y mover las estructuras con cautela, ya que los bordes óseos con una mala manipulación del tejido pueden lacerar alguna estructura.

\section{CONCLUSIONES}

El abordaje transmaxilar de órbita con cantolísis bilateral provee una excelente visualización de todas las estructuras orbitarias, que permite a quien desee comprender con detalle la anatomía de órbita, una alternativa óptima para el aprendizaje a cabalidad de las relaciones topográficas de este espacio, siendo posible realizarlo con un equipo de disección básico y lentes de aumento, sin necesidad de realizar una craneotomía. Sin embargo, debe reservarse únicamente este abordaje para el estudio anatómico de la órbita, ya que para los abordajes quirúrgicos existen en la actualidad abordajes más seguros y con resultados satisfactorios para cada patología.

\section{AGRADECIMIENTOS}

Al Departamento de Anatomía de la Facultad de Medicina de la Universidad de Costa Rica y a la Maestría en Ciencias Morfológicas de este centro por permitir la realización de este estudio y al Sr. Brian Brenes por la producción fotográfica que acompañó este estudio.

\section{REFERENCIAS}

1. Marchal JC. How to perform approaches of the orbit. In: Sindou M. Practical Handbook of Neurosurgery. $1^{\text {st }}$ ed. Vienna: Springer; 2009.

2. Lisowski FP. A guide to dissection of the human body.2nd ed. World Scientific Publishing Co; 2004.

3. Cheung N, McNab AA. Venous Anatomy of the Orbit. Invest. Ophtalmol. Vis. Sci. 2003; 44 (3): 988-995.

4. Dutton JJ. Orbital Surgery. En: Yanoff M, Duker JS. Ophtalmologhy. $3^{\text {rd }}$ ed. Elsevier; 2009.

5. WalterWL. Early surgical repair of blowout fracture of the fracture of the orbital floor by using the transantral approach. South Med J 1972; 65(10): 1229-43.

6. Liss J, Stefko ST, Chung WL. Orbital Surgery: State of the art. Oral Maxillofacial SurgClin N Am 2010; 22: 59-71.

7. Gönül E, et al. Transmaxillary approach to the orbit: An anatomic study. Neurosurgery 2003; 53: 935942.

8. McMains KC, Kountakis SE. Conventional and endoscopic approaches to the orbit and optic nerve. En:Stucker FJ, et al. Rhinology and Facial Plastic Surgery. $1^{\text {st }}$ ed. Springer Berlin Heidelberg; 2009. 
9. Cockerman KP, BejjaniGk, et al. Surgery for orbital tumors. Part II: transorbital approaches. Neurosurg Focus. 2001; 10:E3.

10. Macbeth R. Caldwell Luc and their operation. Laryngoscope 1971; 81:1652-7.

11. Lam KH, Lau WF, et al. Maxillary swing approach to the orbit. Head \& Neck 1991; 13: 107-113.

12. Kosaka M, et al. Novel strategy for orbital tumor resection: surgical "displacement" into the maxillary cavity. Journal of Craniofacial Surgery 2006; 17: 1251-1258.

13. Moore CC, et al. Endoscopic Anatomy of the Orbital Floor and maxillary sinus. Journal of Craniofacial Surgery 2008; 19: 271-276.

14. Farweel DG, Strong EB. Endoscopic repair of orbital floor fractures. Otolaryngologic Clinics of North America 2007; 40: 319-328.

15. Roche PH, Fournier HD, Laccourrete L, Mercier P. Surgical anatomy of the infratemporal fossa using the transmaxillaryapproach. Surg Radiol Anat 2001; 23: 209-213.

16. Breinbauer $\mathrm{K}$ Hayo, Contreras $\mathrm{R}$ José Miguel, Namoncura Carlos. Técnica de Caldwell-Luc en los últimos 16 años: Revisión de sus indicaciones. Rev. Otorrinolaringol. Cir. Cabeza Cuello 2008; 68(3): 247-254.

\section{INFORMACION DE AUTOR:}

Rodríguez Vargas, Gabriel
E-mail: rvgabo@gmail.com

Médico cirujano, Departamento de Anatomía, Universidad de Costa Rica. 